\title{
A pseudotype baculovirus expressing the capsid protein of foot-and-mouth disease virus and a T-Cell immunogen shows enhanced immunogenicity in mice
}

Yimei Cao, Zengjun Lü, Pu Sun, Yuanfang Fu, Feipeng Tian, Xiaofang Hao, Huifang Bao, Xiangtao Liu, Zaixin Liu*

\begin{abstract}
Background: Foot-and-mouth disease (FMD) is a highly contagious disease of livestock which causes severe economic loss in cloven-hoofed animals. Vaccination is still a major strategy in developing countries to control FMD. Currently, inactivated vaccine of FMDV has been used in many countries with limited success and safety concerns. Development of a novel effective vaccine is must.
\end{abstract}

Methods: In the present study, two recombinant pseudotype baculoviruses, one expressing the capsid of footand-mouth disease virus (FMDV) under the control of a cytomegalovirus immediate early enhancer/promoter (CMV-IE), and the other the caspid plus a T-cell immunogen coding region under a CAG promoter were constructed, and their expression was characterized in mammalian cells. In addition, their immunogenicity in a mouse model was investigated. The humoral and cell-mediated immune responses induced by pseudotype baculovirus were compared with those of inactivated vaccine.

Results: Indirect immunofluorescence assay (IFA) and indirect sandwich-ELISA (IS-ELISA) showed both recombinant baculoviruses (with or without T-cell epitopes) were transduced efficiently and expressed target proteins in BHK-21 cells. In mice, intramuscular inoculation of recombinants with $1 \times 10^{9}$ or $1 \times 10^{10} \mathrm{PFU} / \mathrm{mouse}$ induced the production of FMDV-specific neutralizing antibodies and gamma interferon (IFN- $\gamma$ ). Furthermore, recombinant baculovirus with T-cell epitopes had better immunogenicity than the recombinant without T-cell epitopes as demonstrated by significantly enhanced IFN- $\gamma$ production $(P<0.01)$ and higher neutralizing antibody titer $(P<$ 0.05). Although the inactivated vaccine produced the highest titer of neutralizing antibodies, a lower IFN- $\gamma$ expression was observed compared to the two recombinant pseudotype baculoviruses.

Conclusions: These results indicate that pseudotype baculovirus-mediated gene delivery could be a alternative strategy to develop a new generation of vaccines against FMDV infection.

\section{Background}

Foot-and-mouth disease (FMD) is a highly contagious disease of cloven-hoofed animals. The causative agent is foot-and-mouth disease virus (FMDV) which belongs to the genus Aphthovirus in the family Picornaviridae [1]. Foot-and-mouth disease is a major hindrance to

\footnotetext{
*Correspondence: luzengjun920@126.com; liukey@public.lz.gs.cn Lanzhou Veterinary Research Institute of Chinese Academy of Agriculture Science, State Key Laboratory of Veterinary Etiological Biology, National Footand-Mouth Disease Reference Laboratory, Key Laboratory of Animal Virology of Ministry of Agriculture, Xujiaping No.1, Yanchangpu, Lanzhou, Gansu,
} 730046, PR China international trade in animals and animal products. Prevention and eradication of this disease in one country requires sustained effort at significant cost. Vaccination is still a major strategy in developing countries to control FMD. Current FMDV vaccines are available in the form of BEI inactivated antigen in oil adjuvant or aluminum hydroxide and saponin adjuvant [2]. Although these vaccines can induce humoral protective immunity, there are a number of disadvantages with their use, including the inability to differentiate vaccinated from unvaccinated animals, the short-term nature of

\section{Biomed Central}


protection, the extra cost of containment facilities required for their preparation, and the risk of escaped virus $[3,4]$. Thus, it is crucial to develop alternative vaccines.

Since Hofmann reported that recombinant baculovirus containing the cytomegalovirus immediate-early promoter (CMV-IE) was able to drive the expression of a reporter gene in human hepatocytes, baculovirus with a strong mammalian promoter has been used as a novel vector to transfer and express foreign genes in mammalian cells for vaccine development [5-7]. This vector was also shown to be capable of carrying large inserts and infecting a variety of cell lines without any apparent viral replication or cytopathic effects, even at a high multiplicity of infection (MOI) $[7,8]$. Furthermore, it has been reported that a pseudotype baculovirus displaying the glycoprotein of vesicular stomatitis virus (VSV-G) on the envelope can extend the host range, increase the transduction efficiency, and prolong the baculovirusmediated expression in mammalian cells $[9,10]$.

The use of baculovirus as a vector for vaccination was initially described by Aoki and coworkers, who demonstrated that injecting mice with a recombinant vector expressing pseudorabies virus glycoprotein B elicited a measurable humoral response directed against this viral glycoprotein [11]. More recently, direct vaccination with recombinant pseudotype baculovirus induced high-level humoral and cell-mediated immunity against various antigens such as influenza virus HA [12], porcine reproductive and respiratory syndrome virus (PRRSV) [13], Japanese encephalitis virus (JEV) [14], porcine circovirus type 2 (PCV2) [15], Toxoplasma gondii [16], and Plasmodium falciparum [17].

Although it is generally accepted that protective immunity to FMDV is principally due to a neutralizing antibody, a T-cell response is quite clearly necessary for effective immunity; this was demonstrated in pigs that showed no consistent humoral immune response after inoculation with inactivated vaccine but could still resist virulent virus challenge. It is now believed that cellmediated immunity is crucial for protection against FMD. Helper T (Th) lymphocyte epitopes with conserved sequences among different FMDV isolates, and that are recognized by a wide spectrum of MHC Class II alleles in different host species, hold great potential for vaccine design. Residues 20-34 in the structural protein VP4 $[18,19]$ and T-cell epitopes identified on the FMDV non-structural proteins 3D [20,21] and 3A [18] are highly interspecies MHC-restricted Th lymphocyte epitopes. Such epitopes have the additional advantage of being recognized in a heterotypic manner by $\mathrm{T}$-cells of different individuals. The potential of such Th epitopes to improve immunogenicity of a new FMDV vaccine is an ongoing focus of investigation.
Based on these observations, a T-cell epitope fragment was designed with two universal T-cell epitopes and several conservative T-cell epitopes on the FMDV structural and non-structural proteins. Two recombinant pseudotype baculoviruses encoding P12A and 3C, with or without insertion of the above T-cell epitopes, were constructed and their expression was characterized in mammalian cells. In addition, their immunogenicity in a mouse model was investigated. The humoral and cellmediated immune responses induced by pseudotype baculovirus were compared with those of inactivated vaccine. The results obtained further demonstrate that significant cell-mediated immunity to target antigens can be elicited upon injection of recombinant pseudotype baculovirus.

\section{Materials and methods}

Virus and cultured cells

O-serotype FMDV (strain O/HN/CHA/93 with high homology to O/GD/CHA/86, GenBank AJ131468) was provided by the National Foot-and-Mouth Disease Reference Laboratory of China to clone the P1-2A and $3 \mathrm{C}$ genes of $\mathrm{O}$ type FMDV. The BHK-21 cells were grown and maintained in Dulbecco's modified Eagle's medium (DMEM, Invitrogen) supplemented with 10\% heat-inactivated fetal bovine serum (FBS). Spodoptera frugiperda 9 (Sf-9) insect cells were maintained at $27^{\circ} \mathrm{C}$ in Sf-900 II SFM (Invitrogen) supplemented with 2.5\% fetal bovine serum.

\section{Construction of recombinant pseudotype baculovirus}

In order to construct a pseudotype baculovirus, a DNA fragment was synthesized that contained a coding region of a truncated VSV-G, a herpes simplex virus-thymidine kinase (HSV tk) Poly(A) terminal sequence, and a CMV-IE promoter flanked with $B a m \mathrm{H}$ I and $X b a$ I restriction sites. The truncated VSV-G includes a 21 amino acid ectodomain with transmembrane and cytoplasmic tail domains (VSV-GED) as described by Kaikkonen et al. [22]. The above DNA fragment was synthesized by GenScript Corporation (Centennial Ave., Piscataway, NJ 08854, U.S.) with codon optimization for expression in insect cells. This DNA fragment was then inserted into pFastBac Dual vector (Invitrogen) under the control of the polyhedron $(\mathrm{PH})$ promoter via $\mathrm{BamH}$ $\mathrm{I}$ and $\mathrm{Xba}$ I restriction sites. This vector was designated pFastBacDual-GED. The DNA fragment containing the P12A3C expression cassette of O-type FMDV was inserted into pFastBacDual-GED via $\mathrm{Xba}$ I and Hind III restriction sites, resulting in the recombinant transfer plasmid pFastBacDual-GED-P12A3C (Figure 1).

To express a T-cell epitope region in mammalian cells, we utilized the CAG promoter, a composite promoter consisting of the CMV IE enhancer and chicken $\beta$-actin 


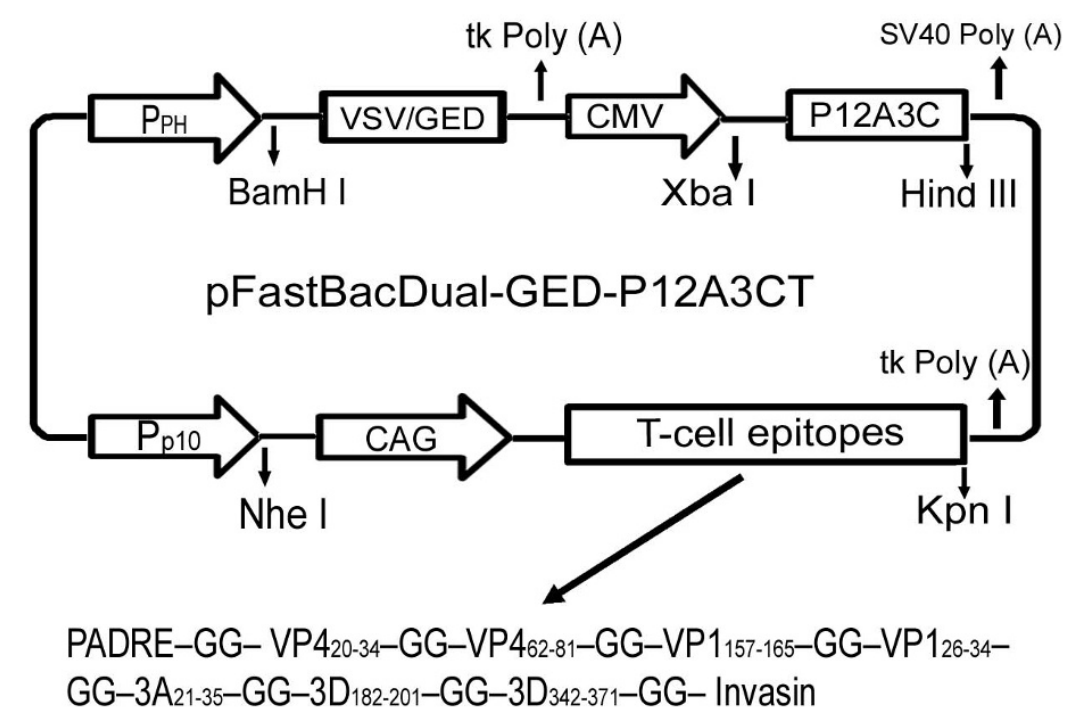

Figure 1 Schematic diagram of the transfer plasmid for construction of recombinant baculovirus. The structure of the transfer plasmid based on pFastBac Dual is displayed. pH, polyhedrin promoter of baculovirus; p10, p10 promoter of baculovirus; VSV/GED, truncated glycoprotein of vesicular stomatitis virus including 21 amino acid ectodomain with transmembrane and cytoplasmic tail domains; CMV, cytomegalovirus immediate-early promoter/enhancer; SV40 or tk poly (A), polyadenylation signal from Simian virus 40 or tk gene of Herpes simplex virus; P12A3C, the P12A3C gene encoding structural and non-structural proteins necessary for the formation of FMDV empty capsid; CAG, a composite promoter consisting of the CMV IE enhancer and chicken $\beta$-actin promoter; T-cell epitopes including a pan DR epitope sequence (PADRE), seven T cell epitope regions in structural or non-structural proteins of FMDV and a invasin immunostimulatory sequence taken from Yersinia, as described by Wang et al. (2002).

promoter [7]. The CAG promoter (737 bp) and a T-cell epitope region (495 bp) were synthesized by GenScript Corporation and inserted into pFastBacDual-GEDP12A3C downstream of the p10 promoter followed by the HSV tk Poly (A) tail. The result was the recombinant transfer plasmid pFastBacDual-GED-P12A3CT. The Tcell epitopes synthesized contained two universal immunostimulatory elements and several T-cell epitopes on structural and non-structural proteins of FMDV (Table 1).

The recombinant baculoviruses Bac-GED, Bac-GEDP12A3C, and Bac-GED-P12A3CT were subsequently

Table 1 T-cell epitopes included in the synthesized T-cell immunogen

\begin{tabular}{|c|c|c|}
\hline Epitopes & Sequence & Function \\
\hline PADRE & AKFVAAWTLKAAA & $T_{H}$ \\
\hline $\mathrm{VP}_{20-34}$ & SIINNYYMQQYQNSM & $\mathrm{T}_{\mathrm{H}}$ \\
\hline VP4 $62-81$ & TQNNDWFSKLASSAFSGLFG & $\mathrm{T}_{\mathrm{H}}$ \\
\hline$V P 1_{157-165}$ & RTLPTSFNY & Tc \\
\hline$V P 1_{26-34}$ & RRQHTDVSF & Tc \\
\hline $3 \mathrm{~A}_{21-35}$ & AAIEFFEGMVHDSIK & $\mathrm{T}_{\mathrm{H}}$ \\
\hline $3 D_{182-201}$ & VDVLPVEHILYTRMMIGRFC & $\mathrm{T}_{\mathrm{H}}$ \\
\hline $3 D_{342-371}$ & WASDYDLDFEALKPHFKSLGQTITPADKS & $\mathrm{T}_{\mathrm{H}}$ \\
\hline Invasin & TAKSKKFPSYTATYQF & $\mathrm{T}_{\mathrm{H}}$ \\
\hline
\end{tabular}

The T-cell epitopes were ligated sequentially via two glycines (Gs) as following: PADRE-GG-VP4 $20-34^{-G G-V P 4}{ }_{62-81}-\mathrm{GG}-\mathrm{VP} 1_{157-165}-\mathrm{GG}-\mathrm{VP1} 1_{26-34}-\mathrm{GG}-3 \mathrm{~A}_{21}$ ${ }_{35}-\mathrm{GG}-3 \mathrm{D}_{182-201}-\mathrm{GG}-3 \mathrm{D}_{342-371}$-GG- Invasin. generated using the Bac-to-Bac System (Invitrogen). Briefly, the recombinant plasmids were transformed into Escherichia coli DH10Bac (Invitrogen), in which all the expression cassettes between Tn7R and Tn7L had been transferred from pFastBacDual-GED, pFastBacDualGED-P12A3C, and pFastBacDual-GED-P12A3CT to the bacmid by site-specific transposition. The subsequent steps for bacmid isolation, transfection, and selection of the recombinant viruses were performed according to the manufacturer's instructions for the Bac-to-Bac System. Recombinant baculoviruses were amplified further by propagation in Sf-9 cells. Virus purification was performed as described previously [23] and purified virus was resuspended in phosphate-buffered saline (PBS, $\mathrm{pH}$ 7.4). The virus titer was determined by the BacPAK Rapid Titer assay (Clontech, Mountain View, CA, USA) in Sf-9 cells.

\section{Baculovirus transduction and protein expression assay}

The BHK-21 cells were seeded at a concentration of $2.5 \times 10^{5}$ cells/well into six-well tissue culture plates (Nunc) until the cells reached approximately $70-80 \%$ confluence. Culture medium was removed and cells were washed three times with PBS ( $\mathrm{pH}$ 7.4). The cells were then incubated in media containing baculovirus for $6 \mathrm{~h}$ at $27^{\circ} \mathrm{C}$. After removal of virus, fresh medium was added and cultures were incubated at $37^{\circ} \mathrm{C}$. At $48 \mathrm{~h}$ 
post-transduction, cells were analyzed for expression of FMDV proteins by the indirect immunofluorescence assay (IFA) and indirect sandwich-ELISA (IS-ELISA). Briefly, cells were fixed with $4 \%$ paraformaldehyde and processed for indirect immunofluorescence assay (IFA) using rabbit serum against FMDV $146 \mathrm{~S}$ antigen, followed by fluorescein isothiocyanate-conjugated goat anti-rabbit IgG. The unbound fluorescent antibodies were washed away with PBST and the cells were sealed with glycerol and observed under a fluorescence microscope. Indirect sandwich-ELISA was performed as described previously [24]. Expression of the VSV-GED protein was monitored in $\mathrm{Sf}-9$ cells by IFA with a rabbit monoclonal antibody specific for VSV-G (Sigma), and the recombinant baculovirus Bac-P12A3C constructed previously was used as a mock-infected control [24].

\section{Immunization of mice}

Six to eight weeks old female BALB/c mice, purchased from the Animal Center, Lanzhou Institute of Biological Products, Gansu Province, 730046, China, were randomly divided into seven groups with eight mice per group. Two groups were injected intramuscularly with $1 \times 10^{9} \mathrm{PFU}$ and $1 \times 10^{10} \mathrm{PFU}$ of Bac-GED-P12A3C. Two groups were injected intramuscularly with $1 \times 10^{9}$ and $1 \times 10^{10}$ PFU of Bac-GED-P12A3CT. The other three groups were immunized intramuscularly with $1 \times 10^{10}$ PFU of Bac-GED, $100 \mu \mathrm{L}$ of PBS, or $100 \mu \mathrm{L}$ inactivated vaccine. Booster immunization was performed identically 3 weeks later. Serum samples were collected from the retro-orbital plexus at 3 and 7 weeks after immunization for serological tests. At 7 weeks after the primary immunization, mice were sacrificed and splenocytes were harvested for IFN- $\gamma$ assay. All animal studies were approved by the Review Board of Lanzhou Veterinary Research Institute, Chinese Academy of Agricultural Sciences (Permission number: SYXK-GAN-2004-0005). The mice in this work were kindly bred during the experiment and mercy-killed at the end of the experiments to reduce the suffering at the most extent.

\section{Detection of specific neutralizing antibodies against FMDV}

Serum samples were heat-inactivated for $45 \mathrm{~min}$ at $56^{\circ} \mathrm{C}$ before testing. All sera were analyzed for neutralizing antibody titers by using a micro-neutralization assay with monolayers of BHK-21 cells [24]. Double dilutions of sera were reacted with $100 \mathrm{TCID}_{50}$ of FMDV O/HN/ $\mathrm{CHA} / 93$ at $37^{\circ} \mathrm{C}$ for $1 \mathrm{~h}$. Cells were then added as indicators of residual infectivity. The microplates were incubated at $37^{\circ} \mathrm{C}$ for 3 days prior to fixation and staining. The endpoint titers were calculated as the reciprocal of the last serum dilution to neutralize 100 TCID $_{50}$ homologous FMDV in $50 \%$ of the wells.

\section{IFN $-\gamma$ release assay}

Mouse splenocytes were isolated as described previously [25]. Splenocytes $\left(1 \times 10^{6} / \mathrm{ml}\right)$ were cultured in 24-well plates at $37^{\circ} \mathrm{C}$ in the presence of $5 \% \mathrm{CO}_{2}$, in the presence or absence of $20 \mu \mathrm{g} / \mathrm{ml}$ FMDV $146 \mathrm{~S}$ antigen. After $20 \mathrm{~h}$ incubation, culture supernatant was harvested and the presence of IFN- $\gamma$ was tested using a commercial mouse IFN- $\gamma$ immunoassay ELISA kit (BD Bioscience) according to the manufacturer's instructions. The concentrations of IFN- $\gamma$ in the samples were determined from the standard curves.

\section{Statistical analysis}

Student's t-test was used to compare the humoral and cellular immune responses between the different groups. $P$-values of $<0.05$ were considered statistically significant.

\section{Results}

\section{Construction and identification of recombinant transfer} plasmid

A schematic diagram of the transfer plasmid for construction of recombinant pseudotype baculovirus is illustrated in Figure 1. The recombinant transfer plasmids pFastBacDual-GED-P12A3C and pFastBacDual-GEDP12A3CT were confirmed by restriction digestion, PCR, and sequence analysis, and there were no mutations introduced in target genes.

For construction of the T-cell immunogen, two universal T-cell epitopes were chosen, a pan HLA DRbinding peptide (PADRE) [26] and an invasin immunostimulatory sequence taken from Yersinia (Invasin) [27]. Eight conservative T-cell epitopes within the structural and non-structural proteins of O/HN/CHA/93 FMDV [28] were linked in tandem by two glycine (G) residues according to the order in Table 1 . The DNA sequence of the designed T-cell immunogen (as shown in Table 1) was synthesized by GenScript Incorporation (http:// www.genscript.com) according to the most commonly occurring codons in mammalian cells.

\section{Expression of the target proteins by recombinant baculoviruses in mammalian cells}

To determine whether Bac-GED-P12A3C and Bac-GEDP12A3CT contain and express the VSV-GED protein, purified virus was analyzed by IFA with a VSV-GEDspecific monoclonal antibody. Expression of the VSV-GED protein was detected both in the Bac-GEDP12A3C (Figure 2A) and Bac-GED-P12A3CT (Figure 2B) preparation, whereas no VSV-G protein was present in the Bac-P12A3C viral preparation (Figure 2C).

To investigate the transduction efficacy of recombinant baculoviruses and the level of FMDV capsid proteins expression within mammalian cells, BHK-21 cells 

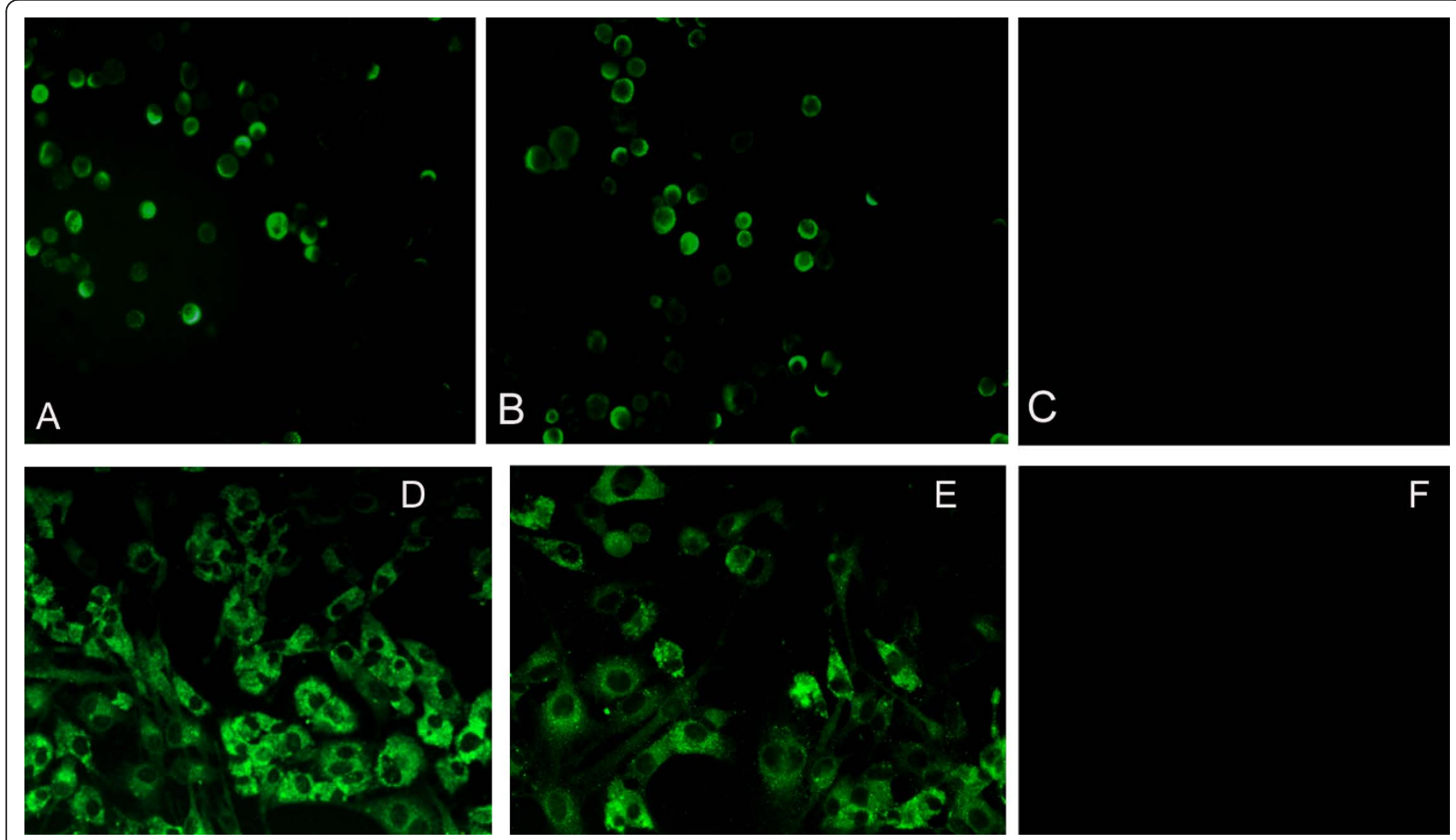

Figure 2 Detection of the VSV-G protein in baculovirus-infected Sf9 cells (A-C) and FMDV capsid proteins in BHK-21 cells (D-F) by immunofluorescence. VSV-G proteins were detected in Sf9 cells infected with baculovirus Bac-GED-P12A3C (A), Bac-GED-P12A3CT (B), or BacP12A3C (C) by staining with a monoclonal antibody against VSV-G. FMDV capsid proteins were detected in BHK-21 cells after infection with baculoviruses Bac-GED-P12A3C (D) and Bac-GED-P12A3CT (E) at $48 \mathrm{~h}$ post-infection by reaction with a rabbit serum against FMDV 1465 antigen. No positive reaction was shown in BHK cells transduced with Bac-GED (F).

were transduced with Bac-GED-P12A3C or BacGED-P12A3CT at a multiplicity of infection (MOI) of 100. An indirect immunofluorescence assay and ISELISA were performed at $48 \mathrm{~h}$ post-transduction to detect the FMDV capsid proteins expressed in BHK-21 cells. As shown in Figure 2D-F, cells transfected with Bac-GED-P12A3C (Figure 2D) and Bac-GED-P12A3CT (Figure 2E) emitted bright fluorescence, but no detectable fluorescence was observed from cells transduced with Bac-GED (Figure 2F). Furthermore, the OD of the harvested cell lysates transfected with Bac-GED-P12A3C and Bac-GED-P12A3CT decreased with greater dilution, but no changes were observed in the BHK-21 cell lysates transfected with Bac-GED (Figure 3).

\section{FMDV-specific neutralizing antibodies elicited by Bac- GED-P12A3C and Bac-GED-P12A3CT in mice}

To determine whether the pseudotype baculovirus expressed proteins could induce FMDV-specific humoral immune responses in vivo, BALB/c mice were immunized intramuscularly with two dosages $\left(1 \times 10^{10}\right.$ and $1 \times 10^{9}$ PFU/mouse) of Bac-GED-P12A3C and BacGED-P12A3CT, while PBS, Bac-GED, and inactivated vaccine were used as controls. Foot-and-mouth disease- specific neutralizing antibodies were monitored at 3 and 7 weeks after the primary immunizations. As shown in Figure 4, mice immunized with $1 \times 10^{10}$ and $1 \times 10^{9}$ PFU of Bac-GED-P12A3C developed mean neutralizing antibody titers of $1: 12$ and $1: 9$ at 3 weeks, respectively, and both increased to $1: 27$ at 7 weeks after primary inoculation. Mice immunized with $1 \times 10^{10}$ and $1 \times 10^{9}$ PFU of Bac-GED-P12A3CT produced mean neutralizing antibody titers of $1: 13$ at 3 weeks which further increased to $1: 35$ and $1: 34$ at 7 weeks after primary inoculation. This was significantly higher than BacGED-P12A3C injected mice $(n=5, P<0.05)$. As expected, sera from mice immunized with Bac-GED or PBS did not display any neutralizing antibody activity throughout the entire duration of study. However, the inactivated vaccine group exhibited a much higher neutralizing antibody titer than any other group $(n=5, P<$ 0.01 ), reaching $1: 16$ at 3 weeks and 1:64 7 weeks after primary inoculation (Figure 4 ).

\section{Cellular immunity elicited by recombinant pseudotype} baculoviruses in mice

To further characterize the cell-mediated immune responses in mice immunized with Bac-GED-P12A3C and 


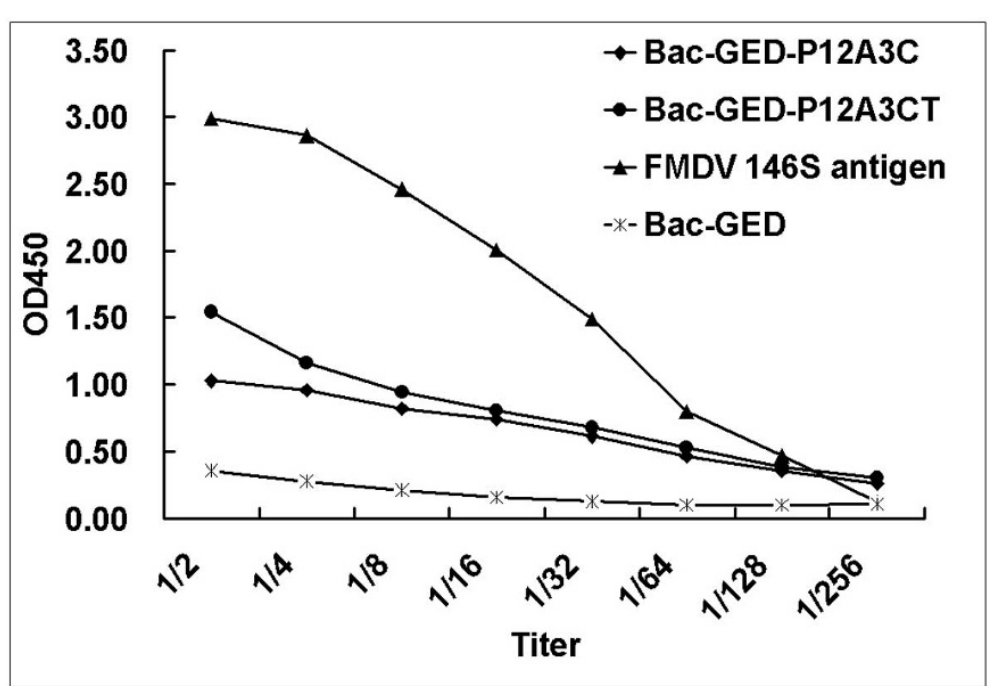

Figure 3 Detection of protein expression in baculovirus-transduced BHK cells by IS-ELISA. Cell lysates from BHK cells transduced with baculovirus were prepared at $48 \mathrm{~h}$ post-transduction and diluted by two-fold serial dilution. The data are presented as the mean of OD450 for each dilution.

Bac-GED-P12A3CT, mice were killed at 7 weeks after primary immunization and IFN- $\gamma$ production in splenocytes was measured by ELISA after restimulation with FMDV $146 \mathrm{~S}$ antigen. As shown in Figure 5, mean IFN- $\gamma$ production reached 1917 and $1332 \mathrm{pg} / \mathrm{ml}$ in mice inoculated with $1 \times 10^{10}$ and $1 \times 10^{9} \mathrm{PFU}$ of Bac-GED-P12A3CT, which was significantly higher than splenocytes from mice that received the same two doses of Bac-GED-P12A3C (771 and $813 \mathrm{pg} / \mathrm{ml}$, respectively) or inactivated vaccine $(667$ $\mathrm{pg} / \mathrm{ml})(n=3, P<0.01)$. As expected, no significant production of IFN- $\gamma$ was detected in PBS-inoculated mice. But splenocytes harvested from baculovirus Bac-GED injected mice produced a relatively higher background non-specific IFN- $\gamma$ response $(450 \mathrm{pg} / \mathrm{ml})$.

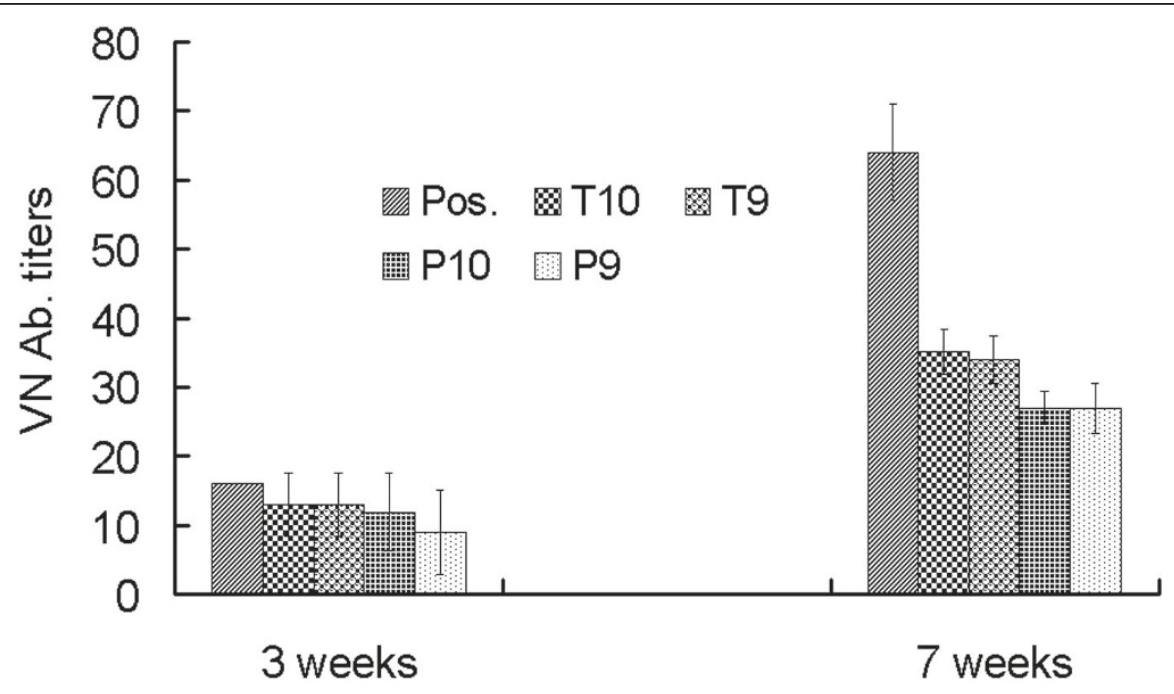

Weeks after vaccination

Figure 4 Neutralizing antibody titers in immunized mice. Neutralizing antibody determinations were performed with sera sampled at 3 and 7 weeks after the primary vaccination. Serum samples were obtained from five mice from each group. Data represent the mean \pm SD. P9, P10 show mice vaccinated with $1 \times 10^{9}$ or $1 \times 10^{10} \mathrm{PFU}$ of Bac-GED-P12A3C; T9, T10 show mice vaccinated with $1 \times 10^{9}$ or $1 \times 10^{10} \mathrm{PFU}$ of Bac-GED-P12A3CT; "inactivated" denotes mice vaccinated with $100 \mu \mathrm{L}$ inactivated vaccine. 


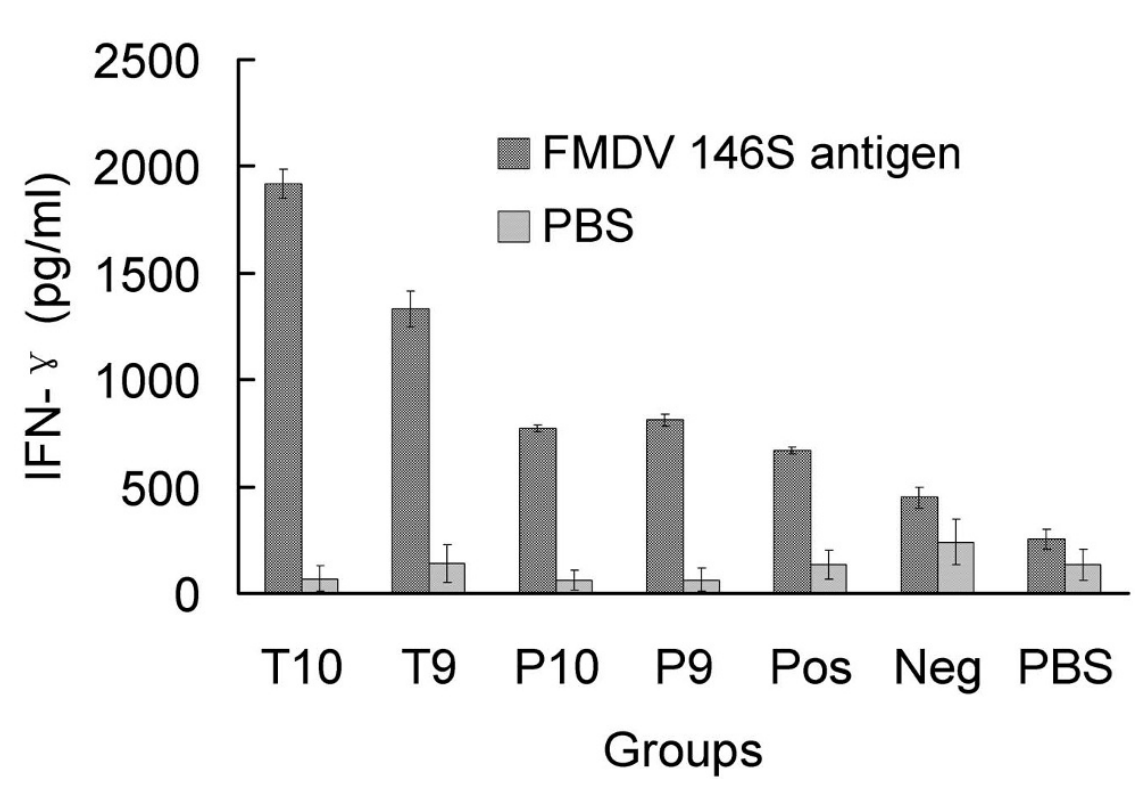

Figure 5 IFN- $\gamma$ production in the supernatant of splenocytes harvested from immunized mice after in vitro restimulation. Mice were immunized as in Figure 4. Splenocytes were isolated 7 weeks after primary immunization and were restimulated in vitro with $146 \mathrm{~S}$ antigen of FMDV for $20 \mathrm{~h}$. IFN- $\gamma$ production in the supernatant was analyzed by ELISA. Data represent the mean \pm SD. Pos, Neg, or PBS denotes mice vaccinated with $100 \mu \mathrm{L}$ inactivated vaccine, $1 \times 10^{10} \mathrm{PFU}$ of Bac-GED, or $100 \mu \mathrm{L}$ of PBS.

\section{Discussion}

Development of a novel vaccine against FMD will have a significant impact on livestock farming and trade. A superior immunization protocol should possess improved immune duration, greater cross protection between different serotypes and genotypes of FMDV, and facilitate the differentiation of vaccinated from infected animals. Recent advances in immunology and molecular biology have stimulated the development of gene-based vaccines. The first is based on the use of plasmid DNA encoding the target antigen of FMDV [29]. However, the use of plasmid DNA as a gene transfer vehicle for immunization is somewhat limited by the low transduction efficiency that hampers its immunogenic potential. To overcome this problem, live virus vector-based vaccine is one possible strategy. Recombinant vaccinia virus and adenovirus for expression of capsid protein has been reported previously [30-32]. Only recombinant adenovirus expressing the empty capsid of FMDV could elicit protective immunity in the host [33-35]. Swine vaccinated with recombinant adenovirus could be protected from virulent virus challenge even with low titers of neutralizing antibody [36]. In the study by Sanz-Parra et al. [32], immunized pigs could develop protective immunity by FMDV-specific T-cell responses but had no detectable neutralizing antibodies. These studies indicated lack of neutralizing antibodies does not necessarily mean absence of protection, at least in pigs, despite the common belief that humoral immunity against FMD is the most important factor determining the efficiency of protection. Thus, the protection observed in these studies was likely mediated by the cellular immune response. In addition, some conserved T-cell epitopes in VP4 and 3A were shown to induce specific immune responses mediated by $\mathrm{CD} 4^{+} \mathrm{T}$ lymphocytes and B lymphocytes, and the higher Th cell response could enhance the production of anti-FMDV neutralizing antibodies [18,37].

Based on these observations, two pseudotype baculoviruses, Bac-GED-P12A3C and Bac-GED-P12A3CT, were constructed to express the empty capsid and a $\mathrm{T}$-cell immunogen with the expectation that such construction could elicit high levels of anti-FMDV neutralizing antibodies. Among these genes, the P1 sequence contains important B-lymphocyte and T-lymphocyte epitopes, allowing stimulation of the cellular and humoral immune responses, and $3 \mathrm{C}$ is a protease necessary for processing of the P12A polyprotein into VP1, VP3 and VP0, the components of self-assembling empty capsids. The T-cell immunogen was designed with two universal T-cell epitopes and several specific T-cell epitopes in the structural and non-structural proteins of FMDV to further enhance the immunogenicity of recombinant pseudotype baculovirus. Both the humoral and cellular immune responses were investigated in a mouse model vaccinated with pseudotype baculoviruses. All mice immunized with various dosages of BacGED-P12A3C and Bac-GED-P12A3CT developed 
detectable neutralizing antibodies, but Bac-GEDP12A3CT infection resulted in a higher antibody response. This indicated that the involvement of the Tcell immunogen could enhance the production of antiFMDV neutralizing antibodies. However, mice inoculated with inactivated vaccine produced much higher titers of neutralizing antibodies than Bac-GED-P12A3C and Bac-GED-P12A3CT, indicating that the amount of capsid protein expressed by pseudotype baculoviruses did not reach that in the inactivated vaccine.

In addition to the humoral immune response, cellmediated immunity has also been suggested to confer protective immunity against FMDV $[32,38,39]$. Host IFN $-\gamma$ production plays a critical role in directing the cell-mediated immune responses for the clearance of intracellular pathogens [40]. Therefore, the production of IFN- $\gamma$ was measured to evaluate the immunogenicity of Bac-GED-P12A3C and Bac-GED-P12A3CT in mice. All mice immunized with various dosages of BacGED-P12A3CT produced much higher levels of IFN- $\gamma$ than mice infected with Bac-GED-P12A3C, indicating that insertion of a T-cell immunogen in Bac-GED-P12A3CT baculovirus could induce a greater cellular immune responses than Bac-GED-P12A3C. Although inactivated vaccine could elicit more neutralizing antibody than either Bac-GED-P12A3C or Bac-GED-P12A3CT, the IFN- $\gamma$ test demonstrated lower levels of cellular immunity. Indeed, mice immunized with Bac-GED-P12A3C and Bac-GEDP12A3CT produced significantly higher levels of IFN- $\gamma$ compared with mice that received inactivated vaccine. One possible reason was that baculovirus vectors can not only transduce mouse skeletal muscle cells, but also efficiently transduce dendritic cells (DCs), the most important APCs [17]. The "adjuvant" effect of baculovirus should also be considered. Baculovirus has the ability to induce innate immune responses through the Toll-like receptor 9 dependent signaling pathway, resulting in the production of various cytokines, including tumor necrosis factor- $\alpha$, IL- 6 , and interferon $[12,23,41,42]$. In this study, a higher background of non-specific IFN- $\gamma$ production was detected from mice injected with Bac-GED recombinant baculovirus, which further suggested the presence of the "adjuvant" effect of baculovirus. A similar result has been observed in pseudotype baculovirus expressing PRRSV's GP5 and M protein [13], JEV's E protein [14], PCV2's ORF2 protein [15], and Toxoplasma gondii SAG1 protein [16].

\section{Conclusion}

In conclusion, Bac-GED-P12A3CT that expressed a Tcell immunogen and FMDV capsid protein had superior immunogenicity to Bac-GED-P12A3C that expressed only the capsid proteins of FMDV, as demonstrated by enhanced IFN- $\gamma$ production and neutralizing antibody titer in mice. The combination of caspid protein and
T-cell epitopes in Bac-GED-P12A3CT recombinant pseudotype baculovirus could be a promising strategy for the development of a new generation of vaccines against FMDV.

\section{Acknowledgements}

This work was supported by Chinese "863" National Programs for High Technology Research and Development (Grant no. 2006AA10A204).

\section{Authors' contributions}

YMC performed most of the experimental work and drafted the manuscript. PS, FPT and XFH participated in the immunization of mice. YFF helped with the ELISA assay. HFB and XTL participated in the analysis of humoral and cellular responses. ZJL and ZXL designed the study, revised the manuscript for important intellectual content and gave final approval of the version to be published. All authors read and approved the final manuscript.

\section{Competing interests}

The authors declare that they have no competing interests.

Received: 24 December 2010 Accepted: 23 February 2011 Published: 23 February 2011

\section{References}

1. Martinez-Salas E, Saiz JC, Davila M, Belsham GJ, Domingo E: A single nucleotide substitution in the internal ribosome entry site of foot-andmouth disease virus leads to enhanced cap-independent translation in vivo. J Virol 1993, 67:3748-3755.

2. Doel TR: FMD vaccines. Virus Res 2003, 91:81-99.

3. Grubman MJ, Baxt B: Foot-and-mouth disease. Clin Microbiol Rev 2004, 17:465-493.

4. The Veterinary Record: FMD outbreak linked to defective drain. Vet Rec 2007, 161:362-363.

5. Hofmann C, Sandig V, Jennings G, Rudolph M, Schlag P, Strauss M: Efficient gene transfer into human hepatocytes by baculovirus vectors. Proc Natl Acad Sci USA 1995, 92:10099-10103.

6. Boyce FM, Bucher NL: Baculovirus-mediated gene transfer into mammalian cells. Proc Natl Acad Sci USA 1996, 93:2348-2352.

7. Shoji I, Aizaki H, Tani H, Ishihi K, Chiba T, Saito I, Myamura T, Matsuura Y: Efficient gene transfer into various mammalian cells, including non-hepatic cells, by baculovirus vectors. J Gen Virol 1997, 78:2657-2664.

8. Fipaldini C, Bellei B, La Monica N: Expression of Hepatitis C virus CDNA in human hepatoma cell line mediated by a hybrid baculovirus-AAV vector. Virology 1999, 255:302-311.

9. Barsoum J, Brown R, McKee M, Boyce FM: Efficient transduction of mammalian cells by a recombinant baculovirus having the vesicular stomatitis virus G glycoprotein. Hum Gene Ther 1997, 8:2011-2018.

10. Hu YC, Tsai $C T$, Chang YJ, Huang JH: Enhancement and prolongation of baculovirus-mediated expression in mammalian cells: Focuses on strategic infection and feeding. Biotechnol Prog 2003, 19:3373-3791.

11. Aoki H, Sakoda Y, Jukuroki K, Takada A, Kida H, Fukusho A: Induction of antibodies in mice by a recombinant baculovirus expressing pseudorabies virus glycoprotein B in mammalian cells. Vet Microbiol 1999, 68:197-207.

12. Abe $\mathrm{T}$, Takahashi $\mathrm{H}$, Hamazaki H, Miyano-Kurosaki N, Matsuura $\mathrm{Y}$, Takaku H: Baculovirus induces an innate immune response and confers protection from lethal influenza virus infection in mice. J Immunol 2003, 171:1133-1139.

13. Wang SP, Fang LR, Fan HY, Jiang YB, Pan YF, Luo Q, Chen HC, Xiao SB: Construction and immunogenicity of pseudotype baculovirus expressing GP5 and M protein of porcine reproductive and respiratory syndrome virus. Vaccine 2007, 25:8220-8227.

14. Li YM, Ye J, Cao SB, Xiao SB, Zhao Q, Liu XQ, Jin ML, Chen HC: Immunization with pseudotype baculovirus expressing envelope protein of Japanese encephalitis virus elicits protective immunity in mice. J Gene Med 2009, 11:57-65.

15. Fan HY, Pan YF, Fang LR, Wang D, Wang SP, Jiang YB, Chen HC, Xiao SB: Construction and immunogenicity of recombinant pseudotype 
baculovirus expressing the capsid protein of porcine circovirus type 2 in mice. J Virol Methods 2008, 150:21-26.

16. Fang R, Feng $H I$, Nie $H$, Wang LX, Tu P, Song QQ, Zhou YQ, Zhao JL: Construction and immunogenicity of pseudotype baculovirus expressing Toxoplasma gondii SAG1 protein in BALB/c mice model. Vaccine 2010, 28:1803-1807.

17. Strauss R, Huser A, Ni S, Tuve S, Kiviat N, Sow PS, Hofmann C, Lieber A: Baculovirus-based vaccination vectors allow for efficient induction of immune responses against Plasmodium falciparum circumsporozoite protein. Mol Ther 2007, 15:193-202.

18. Blanco E, Garcia-Briones M, Sanz-Parra A, Gomes P, De Oliveira E, Valero ML, Andreu D, Ley V, Sobrino F: Identification of T-cell epitopes in nonstructural proteins of foot-and-mouth disease virus. J Virol 2001, 75:3164-3174.

19. Van Lierop MJC, Wagenaar JP, Van Noort JM, Hensen EJ: Sequences derived from the highly antigenic VP1 region 140 to 160 of foot-andmouth disease virus do not prime for a bovine T-cell response against intact virus. J Virol 1995, 69:4511-4514.

20. Collen T, Baron J, Childerstone A, Corteyn A, Doel TR, Flint M, GarciaValcarcel M, Parkhouse RME, Ryan MD: Heterotypic recognition of recombinant FMDV proteins by bovine T-cells: the polymerase (P3Dpol) as an immunodominant T-cell immunogen. Virus Res 1998, 56:125-133.

21. Garcia-Briones MM, Blanco E, Chiva C, Andreu D, Ley V, Sobrino F: Immunogenicity and T cell recognition in swine of foot-and-mouth disease virus polymerase 3D. Virology 2004, 322:264-275

22. Kaikkonen1 MU, Raty JK, Airenne K, Wirth T, Heikura T, Yia-Herttuala S: Truncated vesicular stomatitis virus $G$ protein improves baculovirus transduction efficiency in vitro and in vivo. Gene Ther 2006, 13:304-312.

23. Facciabene A, Aurisicchio L, La Monica N: Baculovirus vectors elicit antigen specific immune responses in mice. J Virol 2004, 78:8663-8672.

24. Cao YM, Lu ZJ, Sun JC, Bai XW, Sun P, Bao HF, Chen YL, Guo JH, Liu XT, Liu ZX: Synthesis of empty capsid-like particles of Asia I foot-and-mouth disease virus in insect cells and their immunogenicity in guinea pigs. Vet Microbiol 2009, 137:10-17.

25. Xiao S, Chen H, Fang L, Liu C, Zhang H, et al: Comparison of immune responses and protective efficacy of suicidal DNA vaccine and conventional DNA vaccine encoding glycoprotein C of pseudorabies virus in mice. Vaccine 2004, 22:345-351.

26. Agadjanyan MG, Ghochikyan A, Petrushina I, Vasilevko V, Movsesyan N, Mkrtichyan M, Saing T, Cribbs DH: Prototype Alzheimer's Disease Vaccine Using the Immunodominant B Cell Epitope from $\beta$-Amyloid and Promiscuous T Cell Epitope Pan HLA DR-Binding Peptide. J Immunol 2005, 174:1580-1586.

27. Wang CY, Chang TY, Walfield AM, Ye J, Shen M, Chen SP, Li MC, Lin YL, Jong MH, Yang PC, Chyr N, Kramer E, Brown F: Effective synthetic peptide vaccine for foot-and-mouth disease in swine. Vaccine 2002, 20:2603-2610.

28. Cooke JN, Westover KM: Serotype-specific differences in antigenic regions of foot-and-mouth disease virus (FMDV): A comprehensive statistical analysis. Infect Genet Evol 2008, 8:855-863.

29. Guo HC, Liu ZX, Sun SQ, Bao HF, Chen YL, Liu XT, Xie QG: Immune response in guinea pigs vaccinated with DNA vaccine of foot-andmouth disease virus O/China99. Vaccine 2005, 23:3236-3242.

30. Abram CC, King AMQ, Belsham GJ: Assembly of foot-and-mouth disease virus empty capsid synthesized by a vaccinia virus expression system. $J$ Gen Virol 1995, 76:3089-3098.

31. Berinstein A, Tami C, Taboga O, Smitsaart E, Carrillo E: Protective immunity against foot-and-mouth disease virus induced by a recombinant vaccinia virus. Vaccine 2000, 18:2231-2238.

32. Sanz-Parra A, Jimenez-Clavero MA, Garcia-Briones MM, Blanco E, Sobrino F, Ley V: Recombinant viruses expressing the foot-and-mouth disease virus capsid precursor polypeptide (P1) induce cellular but not humoral antiviral immunity and partial protection in pigs. Virology 1999, 259:129-134.

33. Mayr GA, Chinsangaram T, Grubman M: Development of replicationdefective adenovirus serotype 5 containing the capsid and $3 C$ protease coding region of foot-and-mouth disease virus as a vaccine candidate. Virology 1999, 263:496-506.

34. Mayr GA, O'Donnell V, Chinsangaram J, Mason PW, Grubman MJ: Immune response and protection against foot-and-mouth disease virus challenge in swine vaccinated with adenovirus-FMDV constructs. Vaccine 2001, 19:2152-2162.
35. Moraes MP, Mayr GA, Mason PW, Grubman MJ: Early protection against homologous challenge after a single dose of replication-defective human adenovirus type 5 expressing capsid proteins of foot-and-mouth disease virus (FMDV) strain A24. Vaccine 2002, 20:1631-1639.

36. Lu ZJ, Bao HF, Cao YM, Sun P, Guo JH, Li PH, Bai XW, Chen YL, Xie BX, Li D, Liu ZX, Xie QG: Protection of guinea pigs and swine by a recombinant adenovirus expressing $\mathrm{O}$ serotype of foot-and-mouth disease virus whole capsid and 3C protease. Vaccine 2008, 26(suppl):48-53.

37. Blanco E, McCullough K, Summerfield A, Fiorini J, Andreu D, Chiva C, Borras E, Barnett P, Sobrino F: Interspecies major histocompatibility complex-restricted Th cell epitope on foot-and-mouth disease virus capsid protein VP4. J Virol 2000, 74:4902-4907.

38. Höhlich BJ, Wiesmüller KH, Haas B, Gerner W, Correa R, Hehnen HR, Schlapp T, Pfaff E, Saalmüller A: Induction of an antigen-specific immune response and partial protection of cattle against challenge infection with foot-and-mouth disease virus (FMDV) after lipopeptide vaccination with FMDV-specific B-cell epitopes. J Gen Virol 2003, 84:3315-3324.

39. Sanz-Parra A, Vazquez B, Sobrino F, Cox SJ, Ley V, Salt JS: Evidence of partial protection against foot-and-mouth disease in cattle immunized with a recombinant adenovirus vector expressing the precursor polypeptide (P1) of foot-and-mouth disease virus capsid proteins. J Gen Virol 80:671-679.

40. Estcourt MJ, Ramshaw A, Ramsay AJ: Cytokine responses in virus infections: effects on pathogenesis, recovery and persistence. Curr Opin Microbiol 1998, 1:411-418

41. Gronowski AM, Hilbert DM, Sheehan KC, Garotta G, Schreiber RD: Baculovirus stimulates antiviral effects in mammalian cells. J Virol 1999, 73:9944-9951.

42. Hervas-Stubbs S, Rueda P, Lopez L, Leclerc C: Insect baculovirus strongly potentiate adaptive immune responses by inducing type I IFN. J Immunol 2007, 178:2361-2369.

doi:10.1186/1743-422X-8-77

Cite this article as: Cao et al: A pseudotype baculovirus expressing the capsid protein of foot-and-mouth disease virus and a T-Cell immunogen shows enhanced immunogenicity in mice. Virology Journal $20118: 77$

\section{Submit your next manuscript to BioMed Central and take full advantage of:}

- Convenient online submission

- Thorough peer review

- No space constraints or color figure charges

- Immediate publication on acceptance

- Inclusion in PubMed, CAS, Scopus and Google Scholar

- Research which is freely available for redistribution 